\title{
Turbulence Modelling of a Single-Phase Flow Cyclone Gasifier
}

\author{
Pantea Hadi Jafari ${ }^{*}$, J. Gunnar I. Hellström² ${ }^{2}$ B. Rikard Gebart ${ }^{1}$ \\ ${ }^{1}$ Department of Engineering Sciences and Mathematics, Division of Energy Science, Luleå University of Technology, Luleå, \\ Sweden \\ ${ }^{2}$ Department of Engineering Sciences and Mathematics, Division of Fluid and Experimental Mechanics, Luleå University of \\ Technology, Luleå, Sweden \\ Email: *pantea.hadi.jafari@ltu.se
}

How to cite this paper: Hadi Jafari, P., I. Hellström, J.G. and Gebart, B.R. (2017) Turbulence Modelling of a Single-Phase Flow Cyclone Gasifier. Engineering, 9, 779-799. https://doi.org/10.4236/eng.2017.99047

Received: August 31, 2017

Accepted: September 23, 2017

Published: September 26, 2017

Copyright $\odot 2017$ by authors and Scientific Research Publishing Inc. This work is licensed under the Creative Commons Attribution International License (CC BY 4.0).

http://creativecommons.org/licenses/by/4.0/

\begin{abstract}
The current work aims to make a foundation for an engineering design of a cyclone gasifier to be able not only to predict its flow field with a suitable accuracy but also to investigate a large number of design alternatives with limited computer resources. A good single-phase flow model that can form the basis in an Euler-Lagrange model for multi-phase flow is also necessary for modelling the reacting flow inside a cyclone gasifier. The present paper provides an objective comparison between several popular turbulence modelling options including standard $\mathrm{k}-\varepsilon$ and SST with curvature corrections, SSG-RSM and LES Smagorinsky models, for the single-phase flow inside cyclone separators/gasifiers that can serve as a guide for further work on the reacting multi-phase flow inside cyclone gasifiers and similar devices. A detailed comparison between the models and experimental data for the mean velocity and fluctuating parts of the velocity profiles are presented. Furthermore, the capabilities of the turbulence models to capture the physical phenomena present in a cyclone gasifier that affects the design process are investigated.
\end{abstract}

\section{Keywords}

Cyclone Gasifier, Turbulence Modelling, Swirling Flow Pattern, Secondary Flow

\section{Introduction}

Cyclone is a stationary mechanical apparatus to separate solid or liquid particles from a carrier gas by utilizing the centrifugal force. One of the recent advanced applications of this device is to use it for biomass gasification, i.e. cyclone gasifier. In a cyclone gasifier, pulverized biomass fuel is fed together with air tangentially 
along the inside surface of a cyclone geometry [1] [2] [3]. Partial combustion inside the cyclone gasifier generates a high temperature that results in conversion of the powder to a medium heating value gas and possibly also a small char residue. So far, several laboratory-scale studies have been carried out to understand the key concepts of this device [4] [5] [6]. Some attempts have also been done to simulate the flow, heat transfer and chemical reactions [7] [8]. The main problem with the CFD approach in combination with turbulent heat transfer and chemical reactions is that the complexity of the global model makes it difficult to assess the accuracy of individual sub-models. For instance, in the recently published papers [7] [8], the models are based on relatively simple eddy-viscosity turbulence models. Although the agreement between these gasifier models and experiments has been encouraging, one cannot rule out the possibility whether this is an effect of cancellation of errors in the different sub models or not. Therefore, it is the purpose of the present paper to focus on the fluid dynamics modeling of the cold single-phase flow in a cyclone gasifier separately in order to develop a better foundation for continued modeling of the reactant multi-phase flow with particle tracking and phase change, but also to have a better understanding of peculiarities in the gas flow of a cyclone geometry.

The flow pattern inside abiomass cyclone gasifier is quite similar to a cyclone separator. However, since the temperature of walls is quite high (around $900^{\circ} \mathrm{C}$ ), the solid wood particles are converted into char and ash. Cyclone separator modeling was pioneered by Barth [9] and was later improved significantly by Muschelknautz [10]. These semi-empirical models can predict the core swirling velocity, pressure drop and separation efficiency in cyclone separators based on geometrical parameters and frictional factors. However, some of the assumptions in these models about the flow pattern are contradicted by experiments [11]. Boysan et al. [12] were among the first to present numerical simulations of the flow field in a cyclone separator. Since then, computational fluid dynamics (CFD) has been frequently used as a tool to predict more geometrical details and performance estimations of cyclone separators. The earliest works showed that eddy viscosity turbulence models such as standard $k-\varepsilon$ were incapable of accurate prediction of the swirling flow in cyclones [13] [14]. Others later reported an appropriate agreement with experimental data using a more complex Reynolds Stress turbulence model [15] [16]. Moreover, more demanding modeling with large eddy simulations (LES) was found to give an improved agreement with LDA measurements [17] [18] [19] [20] [21].

Viscous flow in a typical reverse-flow cyclone can be divided into two types: primary flows, which include the flow through the inlet, the swirling flow in the cyclone, the boundary layers on the vertical walls, and the exit flow in the vortex finder tube; furthermore, secondary flows, which include a boundary layer at the lid of the cyclone with radial inflow, lip leakage at the entrance to the vortex finder, Görtler vortices superimposed on the primary flow and a precessing vortex core (PVC) [11] [19] [22] [23]. 
The main swirling flow in the cyclone is highly turbulent and three-dimensional. Velocity distributions in the near-wall regions and in the shear region between the downward flow near the wall and the upward flow near the centerline are the most challenging features for the modeler. Typically, the tangential velocity is the largest velocity component, and its profile does not change much with axial position in the cyclone. The tangential velocity profiles are similar to a Rankin vortex: a near loss-free vortex surrounding a core of forced solid body rotation, with its maximum value almost at the radius of the vortex finder tube. The maximum tangential velocity is typically thrice the inlet velocity [11] [23] [24]. The axial velocity is of the same order of magnitude as the superficial inlet velocity. The axial flow is directed downward towards the dust bin in the near wall region, and upwards to the vortex finder tube in the core of the cyclone.

The radial velocity is generally the smallest component, and is more difficult to measure accurately. This component is uniform over the height of the cyclone, except for the greatest inward velocity on the edge of the vortex finder tube opening, generally referred to as "lip leakage". This peak in radial velocity is due to the effect of the secondary flows in the boundary layer of the cyclone lid which can cause slip at the lid leading to non-ideal separation of particles [11].

Apart from the primary flows, secondary flows in the cyclone are produced due to imbalances between the radial pressure gradient from the rapidly rotating flow and inertial forces. There are two phenomena that are of particular importance; the first is the thin boundary layer on the lid of the cyclone that is caused by the no-slip condition at the surface, which means that the centrifugal force diminishes close to the wall while the radial pressure gradient is the same as in the free stream. This in its turn means that there is a net force on the fluid elements that will accelerate the flow inwards in a thin boundary layer [22]. When the boundary layer flow reaches the vortex finder tube it will be forced to flow in the axial direction until it comes to the lip of the vortex finder where it will be entrained by the fast flow going into the vortex finder. The second phenomenon is the possibility for "Taylor-Görtler" flow instabilities caused by the swirling flow in the cylindrical part of the cyclone [22]. Both of these phenomena are unwanted since they will lead to reduced separation efficiency due to carry-over of particles.

Another significant secondary flow phenomenon is the PVC that has been observed in experimental studies [15] [25] [26] [27]. The PVC is a quasi-periodic motion of the main vortex core around the geometrical centerline of the cyclone. Based on numerous experiments, it has been found that cyclones exhibit a distinctly non-axisymmetric and low frequency unstable behavior. Moreover, the vortex core precession about the centerline which is caused by the hydrodynamic instabilities of the flow, enhances velocity fluctuations, and hence, turbulence fluctuations. Therefore, close to the center where the gas goes out through the vortex finder, velocity fluctuation levels are very high. This means that simulations should not only be calculated in terms of average velocities, but also fluctuating velocities are very important. Thus, a high spatial and temporal 
resolution is necessary to adequately resolve the single-phase flow field of the cyclone [28].

Accordingly, turbulence is an inherent characteristic of a cyclone gasifier which has a significant effect on the heat and mass transfer in an Eulerian-Lagrangian frame work. However, in the present work, the aim is to choose a model being able to investigate a large number of design alternatives in as short time as possible with limited computer resources. Hence, the cost-performance is a crucial parameter especially since the turbulence model will be applied to predict the multi-phase reacting flow inside of a cyclone gasifier. Tracking particle paths and solving additional equations for the heterogeneous and homogeneous chemical reactions will considerably lengthen the computational time. An essential pre-requisite of an appropriate turbulence model for the reacting flow inside the cyclone geometry is first to have capability to simulate the single-phase flow. The current work aims to model single-phase turbulent gas flow inside isothermal cyclone separator/gasifier using a number of popular turbulence models and to compare the obtained results with each other and with detailed Laser Doppler Anemometry (LDA) velocity measurements, in the literature [29], which can be considered as a benchmark for future modeling of the reactant multi-phase flow. The turbulence models that have been selected are: 1) standard $\mathrm{k}-\varepsilon$ with curvature correction; 2) Shear stress transport (SST) with curvature correction; 3) SSG Reynolds stress model (SSG-RSM); and 4) large eddy simulation (LES). The comparison between the mean and root-mean-squared simulated velocity profiles and the experimental data is presented here. However, computational cost and the ability of the models to capture the significant phenomenon in a cyclone will be the main focus.

\section{Governing Equations}

The gas flow in a cold flow cyclone gasifier can be treated as incompressible and isothermal. Therefore, the Reynolds-averaged Navier-Stokes (RANS) equations can be written in the following form [30]:

$$
\begin{gathered}
\frac{\partial \bar{u}_{i}}{\partial x_{i}}=0 \\
\frac{\partial \bar{u}_{i}}{\partial t}+\bar{u}_{i} \frac{\partial \bar{u}_{i}}{\partial x_{j}}=-\frac{1}{\rho} \frac{\partial \bar{p}}{\partial x_{i}}+v \frac{\partial^{2} \bar{u}_{i}}{\partial x_{j} \partial x_{j}}-\frac{\partial}{\partial x_{i}}\left(\overline{u_{i}^{\prime} u_{j}^{\prime}}\right)
\end{gathered}
$$

where $\bar{u}$ and $u^{\prime}$ are defined as the mean (time-averaged) and fluctuating components of the fluid velocity, respectively, i.e. $u=\bar{u}+u^{\prime}$. Over barred term, $-\overline{u_{i}^{\prime} u_{j}^{\prime}}$, is known as the Reynolds stress tensor that accounts for turbulent fluctuation in fluid momentum ${ }^{1}$. The objective of RANS-based turbulence models is to calculate the Reynolds stresses. In eddy viscosity based models, the Reynolds stresses are linearly associated to the mean velocity gradients and eddy (turbulent) viscosity as follows [31]:

${ }^{1}$ List of symbols at the end of the paper. 


$$
-\rho \overline{u_{i}^{\prime} u_{j}^{\prime}}=\mu_{t}\left(\frac{\partial \bar{u}_{i}}{\partial x_{j}}+\frac{\partial \bar{u}_{j}}{\partial x_{i}}\right)-\frac{2}{3} \rho k \delta_{i j}
$$

where $\delta_{i j}$ is the Kronecker delta and $\mu_{t}$ is the eddy viscosity that has to be modeled. For the widely used $k-\varepsilon$ models, it is linked to the turbulent kinetic energy $(k)$ and rate of dissipation $(\varepsilon)$ through the relation [31]:

$$
\mu_{t}=C_{\mu} \rho \frac{k^{2}}{\varepsilon}
$$

where $C_{\mu}$ is an empirical constant $(=0.09)$. The SST turbulence model is another two-equation eddy viscosity model used here, which utilizes the $k$ - $\omega$ formulation in the boundary layers of the wall, while it switches to a $k-\mathcal{E}$ behavior to model the free stream [32]. In the $k$ - $\omega$ models, the turbulent viscosity is defined based on the turbulent kinetic energy $(k)$ and turbulent frequency $(\omega)$ :

$$
\mu_{t}=\rho \frac{k}{\omega}
$$

The drawback of the eddy-viscosity models, when considering cyclone flows, is that they are insensitive to swirling flows. Hence, to account for swirling effects, Spalart and Shur [33] derived an empirical modification of eddy-viscosity turbulence models. The empirical function is used as a multiplier of the turbulent production term as follows [33]:

$$
\begin{aligned}
P_{k}=\rho \overline{u_{i}^{\prime} u_{j}^{\prime}} \frac{\partial \rho \bar{u}_{i}}{\partial x_{j}} & =\mu_{t}\left(\frac{\partial \bar{u}_{i}}{\partial x_{j}}+\frac{\partial \bar{u}_{j}}{\partial x_{i}}\right) \frac{\partial \bar{u}_{i}}{\partial x_{j}} \\
P_{k} & \rightarrow P_{k} \cdot f_{r}
\end{aligned}
$$

where $P_{k}$ is the turbulent production term due to the viscous forces, and $f_{r}$ is the empirical function applied to stabilize the rotating flow [33] [34].

The RSM models are based on directly solving the transport equations to obtain all individual stress components [35]. Since the SSG-RSM developed by Speziale et al. [36] has been shown to be more accurate than the other versions of RSM models for most flows, and particularly for swirling flows, it was selected for this study (based on recommendation of [34]). A detailed explanation of both eddy viscosity and RSM models can be found in [37].

An alternative approach to RANS modeling is the LES technique that directly solves for large-scale fluctuating motions with characteristic size greater than the grid size; and implicitly models sub-grid scales (SGS) for eddies that are smaller than the grid size [38]. For this technique, the time-dependent Navier-Stokes equations are filtered and used as the governing equations. For an incompressible flow, the filtered Navier-Stokes equations can be written in the following way:

$$
\begin{gathered}
\frac{\partial \overline{\bar{u}}}{\partial x_{i}}=0 \\
\frac{\partial \overline{\bar{u}}_{i}}{\partial t}+\overline{\bar{u}}_{j} \frac{\partial \overline{\bar{u}}_{i}}{\partial x_{j}}=-\frac{1}{\rho} \frac{\partial \overline{\bar{p}}}{\partial x_{i}}+\frac{\partial}{\partial x_{j}}\left[v\left(\frac{\partial \overline{\bar{u}}_{i}}{\partial x_{j}}+\frac{\partial \overline{\bar{u}}_{j}}{\partial x_{i}}\right)\right]-\frac{\partial \tau_{i j}^{s g s}}{\partial x_{i}}
\end{gathered}
$$


where $\overline{\bar{u}}$ is the filtered fluid velocity; $\overline{\bar{p}}$ is the filtered static pressure; and the $\tau_{i j}^{s g s}$ term is the SGS stress tensor that is defined by:

$$
\tau_{i j}^{s g s}=\overline{\bar{u}}_{i} \overline{\bar{u}}_{j}-\overline{\bar{u}}_{i} \overline{\bar{u}}_{j}
$$

By using the Boussinesq hypothesis [39], the SGS stress is calculated:

$$
-\left(\tau_{i j}^{s g s}-\frac{\delta_{i j}}{3} \tau_{k k}^{s g s}\right)=2 v^{s g s} \overline{\bar{s}}_{i j}^{\text {sgs }}
$$

where $v^{\text {sgs }}$ is the SGS turbulent viscosity; and $\overline{\bar{s}}_{i j}^{\text {sgs }}$ is the rate of strain tensor for the large scale defined by:

$$
\overline{\bar{s}}_{i j}^{s g s}=\frac{1}{2}\left(\frac{\partial \overline{\bar{u}}_{i}}{\partial x_{j}}+\frac{\partial \overline{\bar{u}}_{j}}{\partial x_{i}}\right)
$$

Among three commonly used SGS models, the Smagorinsky model [40], the dynamic Smagorinsky-Lilly [41] [42], and the wall-adapted local eddy-viscosity (WALE), the Smagorinsky model was selected for the present study, since the Smagorinsky model compared to the other models is more simple and stable, and less time-consuming. More details about LES models can be found in [43].

\section{Numerical Setup}

A cylinder-on-cone reverse-flow gas cyclone with tangential inlet, drop tube and dust bin (see Figure 1, Table 1), used in the experimental study by Obermair et al. [29], is considered as the case study.

The 3D-geometry of the considered cyclone was created using ANSYS Design Modeler 14.5. The hexahedral computational grids were generated in ICEM CFD 14.5, using a number of blocks to sub-divide the geometry and mesh the blocks separately. The near wall mesh was optimized depending on the type of wall boundary condition (i.e. wall function or resolved boundary layer) in each case. The simulations were performed using the ANSYS CFX 14.5 commercial solver on a 64-bit cluster computer with 32 cores.

Five grids were produced (Table 2) and used for a grid sensitivity study, using a Richardson extrapolation (RE) method [44] to estimate the errors, using the pressure drop over the cyclone as the evaluation parameter. The mesh convergence study was first performed using the $\mathrm{k}-\varepsilon$ turbulence model for all five meshes in Table 2. The mean tangential and axial velocities for the five cases compared to experimental results are illustrated in Figure 2. As it can be seen from the figure, the differences between the meshes are small, but the agreement with experiments is poor due to the exaggerated diffusivity of the k- $\varepsilon$ model

The pressure recovery coefficient, $C_{P}$, was chosen as the goal function for a formal mesh sensitivity study. $C_{P}$ is defined as follows:

$$
C_{p}=\frac{\frac{1}{A_{\text {out }}} \int_{A_{\text {out }}} P_{\text {out }} \mathrm{d} A-\frac{1}{A_{\text {in }}} \int_{A_{\text {in }}} P_{\text {in }} \mathrm{d} A}{\frac{1}{2} \rho_{g} U_{\text {in }}^{2}}
$$




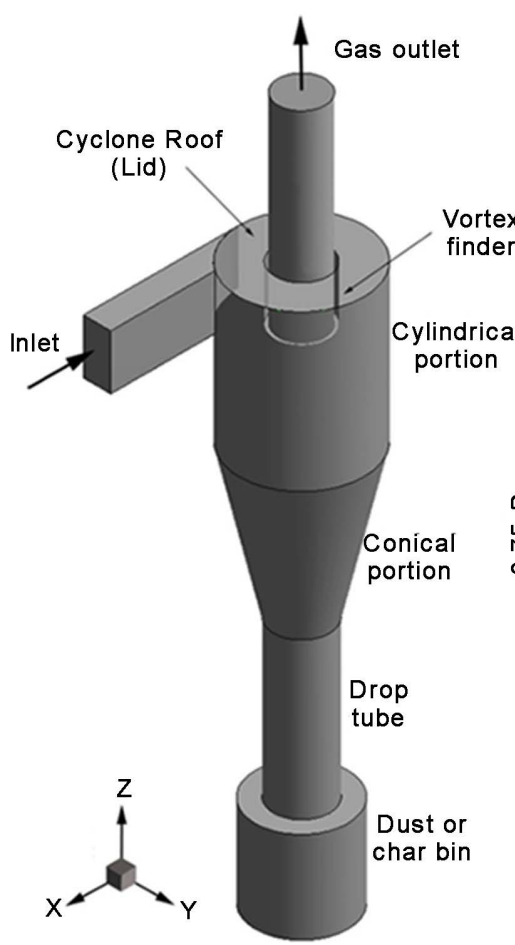

(a)

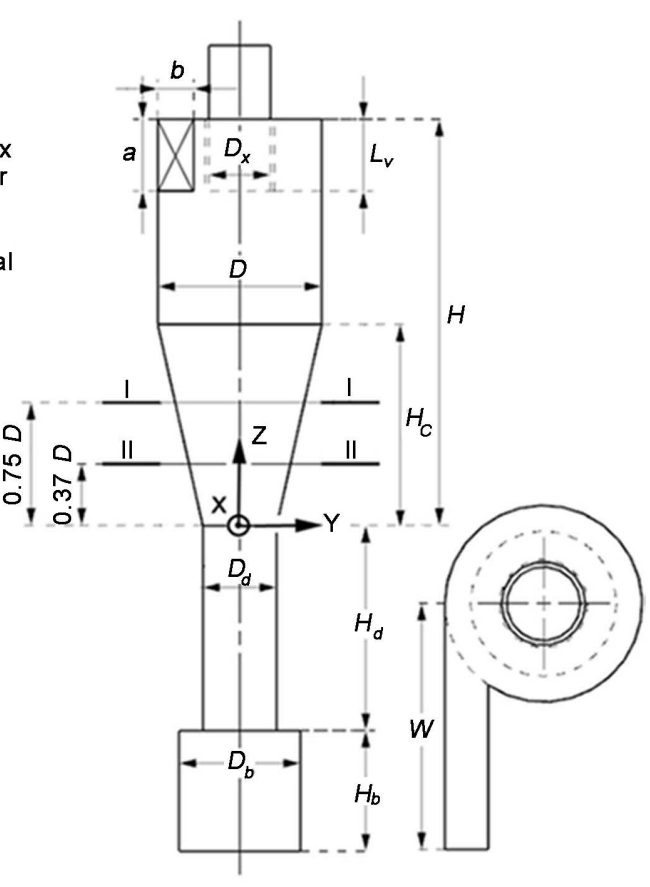

(b)

Figure 1. Geometry and computational domain: (a) 3D view; (b) nomenclature for the simulated geometry: (a), inlet height; (b), inlet width; $D$, body diameter; $D_{x}$, vortex finder diameter; $D_{d}$ drop tube diameter; $D_{b}$, dust bin diameter; $H$, total cyclone height; $H_{\mathcal{C}}$ cone height; $H_{d}$ drop tube height; $H_{b}$, dust bin height; $L_{v}$ vortex finder length; $W$, inlet length.

Table 1. Geometrical dimensions of the cyclone, normalized by the cyclone body diameter $D=0.4 \mathrm{~m}$ (vortex finder wall thickness is $0.01 \mathrm{~m}$ ).

\begin{tabular}{ccccccccccc}
\hline$a / D$ & $b / D$ & $W / D$ & $D_{x} / D$ & $D_{d} / D$ & $D_{b} / D$ & $L_{v} / D$ & $H / D$ & $H_{c} / D$ & $H_{d} / D$ & $H_{b} / D$ \\
\hline 0.44 & 0.22 & 1.25 & 0.38 & 0.45 & 0.74 & 0.44 & 2.48 & 1.23 & 1.25 & 0.74 \\
\hline
\end{tabular}

where $\rho_{g}$ is the gas density; and the subscripts in and out denotes values at the inlet and outlet, respectively. The change of $C_{P}$ with mesh size is shown in Figure 3. It can be seen from Figure 3 that the convergence is monotonic but that the solutions for the two coarsest grids (meshes (1) and (2)) seem to be outside the so called "asymptotic region" where a simple power law will give a good fit to the data. In order to quantify the error, the grid convergence index (GCI) method [44], which is based on Richardson extrapolation, was used. The local apparent order of accuracy $\mathrm{p}$ in the fine-grid solution is 4.18 , and the numerical uncertainty is $0.47 \%$. Assuming that the relative error in $C_{P}$ is representative of the errors in the rest of the variables, this implies that the medium mesh gives an acceptable error for further investigations of other turbulence models.

For the SSG-RSM model also, the three last fine grids were checked, and the results showed the same trend of convergence. However, note that for other 


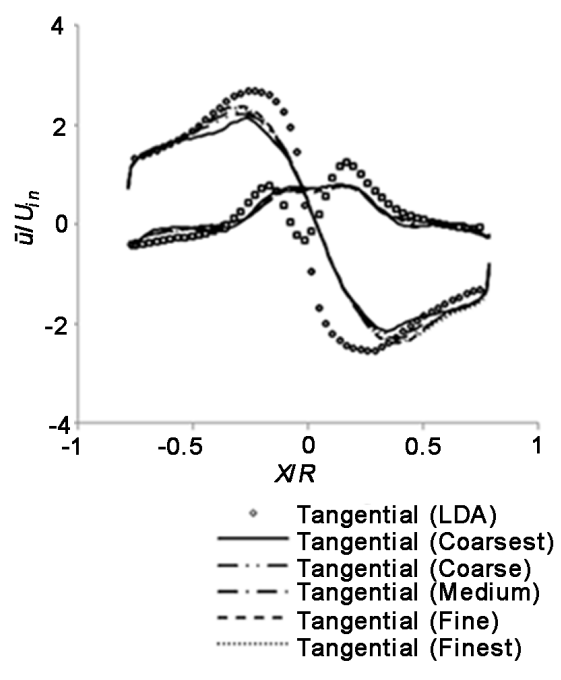

(a)

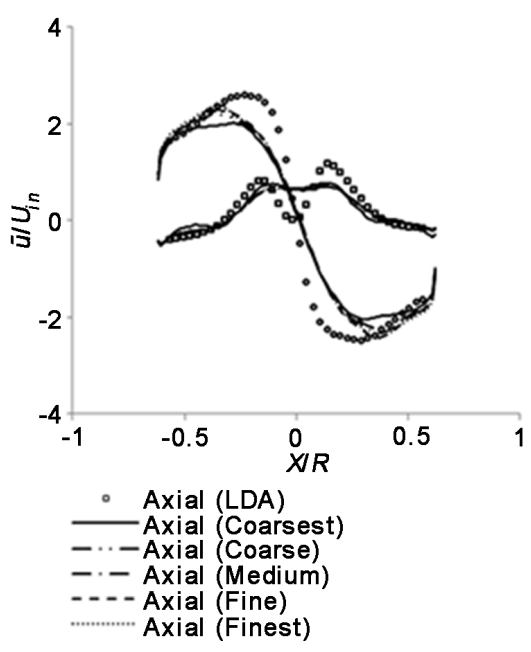

(b)

Figure 2. Comparison of the simulated tangential and axial velocities by the $\mathrm{k}-\varepsilon$ turbulence model for five different grids along two different diameters corresponding to: (a) section I-I, (b) section II-II. The experimental values measured with LDA (from [29]) are shown for comparison.

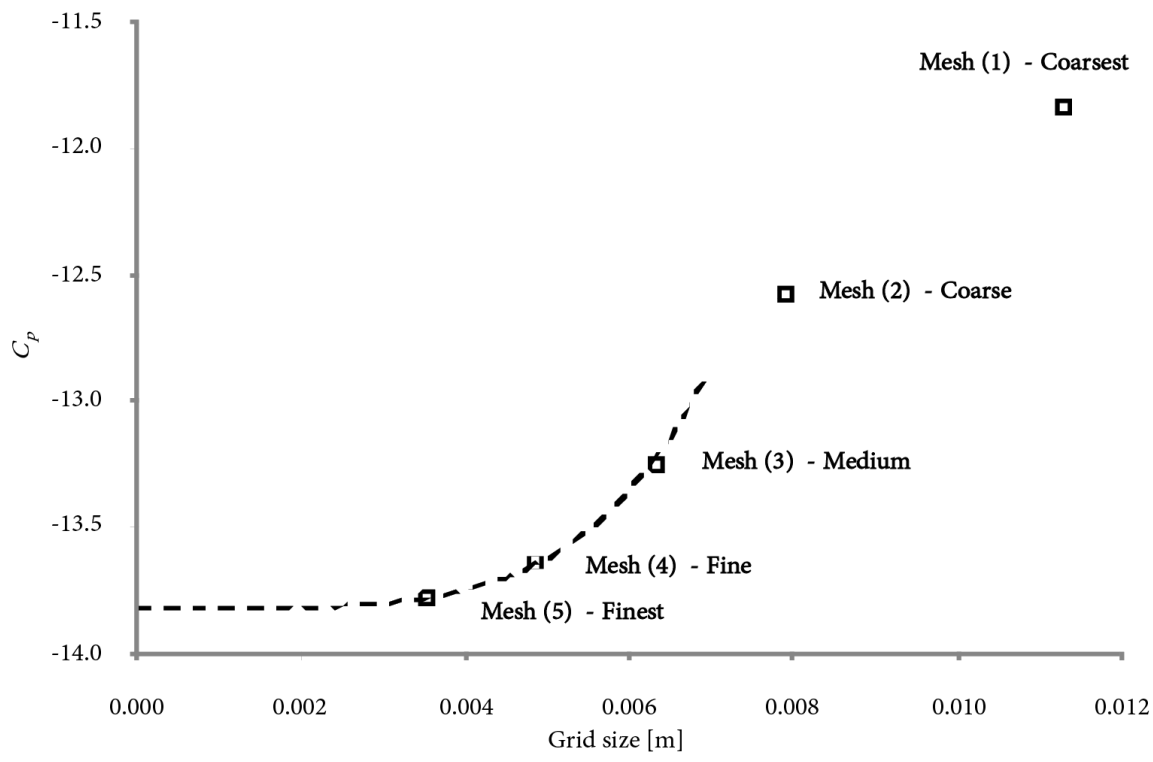

Figure 3. Change of pressure recovery coefficient $\left(C_{p}\right)$ values in different grid resolutions and a power law curve fit to the grids $(3,4$ and 5$)$ using Richardson extrapolation.

Table 2. Characteristics of the CFD grids.

\begin{tabular}{cccccc}
\hline $\begin{array}{c}\text { Mesh } \\
\text { Characteristics }\end{array}$ & $\begin{array}{c}\text { Mesh 1 } \\
\text { (Coarsest) }\end{array}$ & $\begin{array}{c}\text { Mesh 2 } \\
\text { (Coarse) }\end{array}$ & $\begin{array}{c}\text { Mesh 3 } \\
\text { (Medium) }\end{array}$ & $\begin{array}{c}\text { Mesh 4 } \\
\text { (Fine) }\end{array}$ & $\begin{array}{c}\text { Mesh 5 } \\
\text { (Finest) }\end{array}$ \\
\hline $\begin{array}{c}\text { Total number of } \\
\text { nodes }\end{array}$ & 190,095 & 541,059 & $1,043,022$ & $2,297,118$ & 5856,489 \\
$\begin{array}{c}\text { Total number of } \\
\text { elements }\end{array}$ & 181,568 & 524,168 & $1,016,307$ & $2,252,504$ & $5,773,232$ \\
$\begin{array}{c}\text { Representative } \\
\text { grid size }(\mathrm{m})\end{array}$ & 0.011 & 0.008 & 0.006 & 0.005 & 0.004 \\
\hline
\end{tabular}


turbulence models the choice of grid size is somewhat not straightforward, and due to the time limitations it is not possible to check all the turbulence models with all grids. Therefore, the medium grid system is a good choice to start for all simulations.

For the unsteady RANS models, i.e. k- $\varepsilon$, SST and SSG-RSM, the high resolution, and for LES simulation, the unbounded central difference advection scheme was applied; however, first-order upwind was used for solving the turbulence equations in all simulations (as suggested by [45]). The second-order backward Euler transient scheme was selected for all simulations, since it was difficult to get a good converged solution using a direct stationary solution approach. It is most likely that the root cause of it comes from the PVC phenomenon that generates continuously fluctuating forces on the flow field near the center [19].

For the convergence criteria, the residual target was set to $1 \times 10^{-6}$ rootmean-square (RMS) in all cases. To determine the timestep size, an adaptive time stepping technique was used for all simulations by which the timestep is calculated automatically, using a Courant number criterion. This method was necessary, since specifying a fixed size for the timestep created convergence problems; especially for the RANS models (see details in Table 3). At the same time, as shown in Table 3, 3 - 5 iterations within each timestep were implemented for all simulations which means that the applied time stepping method can control the transient behavior of the flow appropriately in all simulations [45].

Furthermore, another important criterion is the dimensionless distance from the first mesh node to the wall (the $y^{+}$value of the first node). The wall function approach and the required $y^{+}$value depend on the flow behavior and the turbulence model being used. Since k- $\varepsilon$ and SSG models use a wall function approach for the near wall region, a maximum distance of the first grid point for all meshes off the wall was adjusted to the range of $y^{+}=40-80$, and a scalable wall function [45] was applied. However, for the SST and LES models, which solve the transport equations also for the inner parts of the boundary layer, the first grid point should be at a position of $y^{+}<2$ [37] and wall function is automatically determined by the software.

At the inlet, a plug flow profile with a normal-direction speed equal to 12.68 $\left(\mathrm{m} \cdot \mathrm{s}^{-1}\right)$ was used as the boundary condition. Also, for all RANS models, a

Table 3. Timestep settings for all simulations.

\begin{tabular}{clcccc}
\hline Turbulence Models & Timestep Adaption & $\begin{array}{c}\text { Max. } \\
\text { Timestep }\end{array}$ & $\begin{array}{c}\text { Min. } \\
\text { Timestep }\end{array}$ & $\begin{array}{c}\text { Max. Coeff. } \\
\text { Loops }\end{array}$ \\
\hline k- $\boldsymbol{E}$ model & Max Courant No.: & 8.0 & 0.01 & $1 \mathrm{e}-05$ & $4-5$ \\
SST model & RMS Courant No.: & 1.0 & 0.01 & $1 \mathrm{e}-05$ & $4-5$ \\
SSG-RSM model & Max Courant No.: & 8.0 & 0.01 & $1 \mathrm{e}-05$ & $4-5$ \\
LES Smagorinsky model & RMS Courant No.: & 0.6 & 0.01 & $1 \mathrm{e}-06$ & 3 \\
\hline
\end{tabular}


medium turbulence level at the inlet with an intensity of 5\% was applied. Additionally, the cyclone was operated with an air density of $1.17\left(\mathrm{~kg} \cdot \mathrm{m}^{-3}\right)$, and a dynamic viscosity of $1.8 \times 10^{-5}$ (Pa.s), corresponding to a temperature of $20\left({ }^{\circ} \mathrm{C}\right)$.

At the gas outlet, the boundary condition for all RANS simulations was set to "opening" type boundary condition. This boundary condition is a Neumann type boundary condition that specifies the normal derivative at the outlet for all variables except the pressure, and therefore, the gas can both exit and enter through the outlet. However, for the LES simulation, the "outlet" boundary condition, in which the code makes artificial walls at the outlet as a barrier for the flow to prevent inflow, was used. In fact, since the flow at the outlet tries to return to the computational domain and some recirculation will occur that causes destabilization of the solution for LES simulation, using an outlet boundary condition therefore increases the robustness of the calculation [45].

During the simulations, some representative quantities such as axial and tangential velocities at certain points in the cyclone body, drop tube and dust bin were monitored. After the flow field became periodic and statistically steady-state (quasi steady), a time-averaging process would be performed over 10,000 time steps. In arithmetic average, timestep-weighted simulated values from the latest timestep are added recursively to the accumulating statistics. Finally, the time-averaged velocity values are compared the LDA measurements.

\section{Results and Discussion}

\subsection{General Impressions of the Flow Field}

A snapshot of the flow-fields from the LES simulation in the $x-Z$ cut plane through the center and the $x-y$ plane at a location very close to the cyclone lid is presented in Figure 4. In Figure 4(a), an instantaneous view of the internal flow-field in the cyclone body, conical part and entrance region of the vortex finder is shown. A close inspection of the results shows that there is a thin boundary layer with radial flow towards the vortex finder at the top of the cyclone (see Figure 4(b)), as is to be expected from a local boundary layer analysis [22]. The boundary layer is deflected in the axial direction when it meets the vortex finder wall and the resulting flow is eventually entrained in the main flow leaving the cyclone at the lip of the vortex finder. Another flow detail that can be observed in Figure 4(a) and Figure 4 (b) is the presence of Taylor-Görtler vortices [23] close to the cyclone body wall. These secondary vortices are generated by a centrifugal instability near the concave wall of the cyclone [22]. Notice that the Taylor-Görtler vortices are superimposed on the much faster tangential flow in the cyclone but at the same time they will have a significant effect on the separation efficiency of the cyclone. From Figure 4(c), it can be seen that vortex core is slightly off-axis within the vortex finder which means that the point of zero circumferential velocity at the top of the cyclone is not exactly on the centerline of the cyclone geometry. This is a sign of the existence of a PVC in the simulations. 


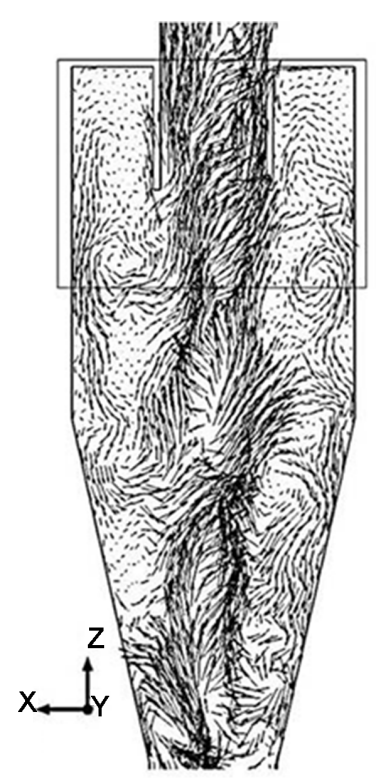

(a)

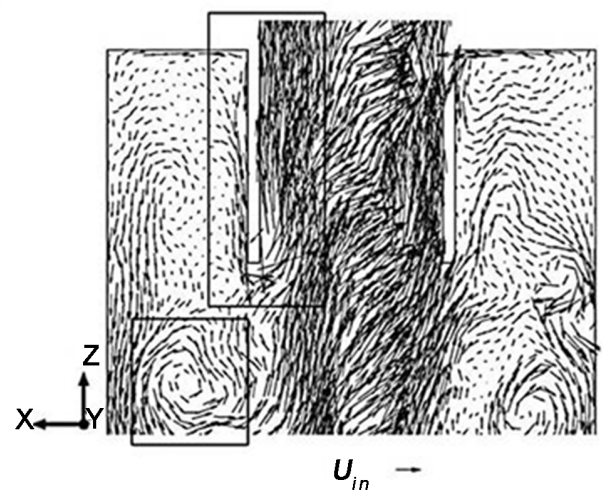

(b)

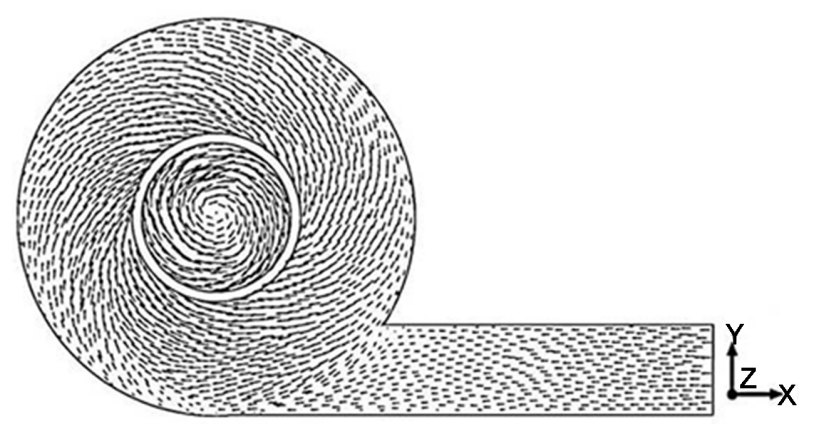

(c)

Figure 4. Flow field from the LES in two cut planes: (a) Instantaneous flow field in the (vertical) $X$-Z cut plane through the center of the cyclone geometry (at $Y=0$ ), (b) detail of the instantaneous flow in the entry region of the vortex finder tube, (c) instantaneous realization of the flow field in a (horizontal) $X$ - $Y$ cut plane at $Z=2.47 D$.

In Figure 5, contour plots of instantaneous velocity values are shown in six cross sections of the body cyclone and conical portion. The results confirm that with increasing the distance from the centerline, the velocity is rising to a maximum value and then decreases towards the wall. Furthermore, signs of the off-axis movement of the core through the centerline can be noticed, which is another proof of the existence of a quasi-periodic motion of the vortex core that is well captured by the LES.

\subsection{Comparison of Turbulence Models}

In order to assess the capability of the turbulence models to represent the peculiarities of the flow field in the cyclone, the mean and RMS values of tangential and axial velocity profiles of single phase CFD simulations and LDA measurement data of [29] have been compared along two diameters in the conical part of the cyclone (shown in Figure 1). 


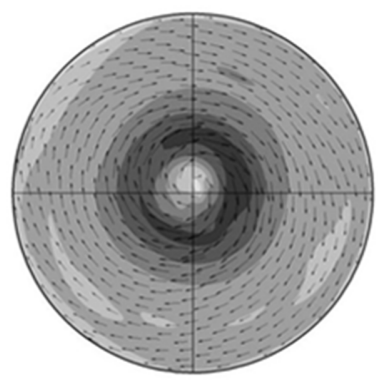

(a)

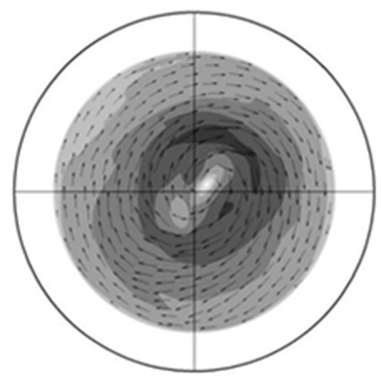

(d)

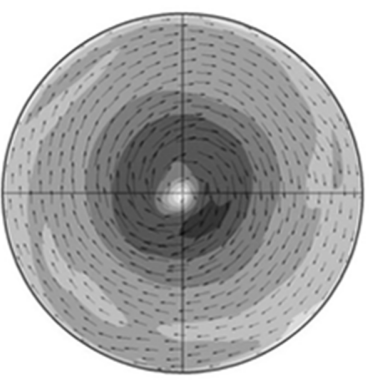

(b)

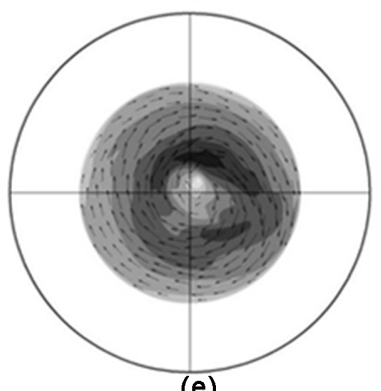

(e)

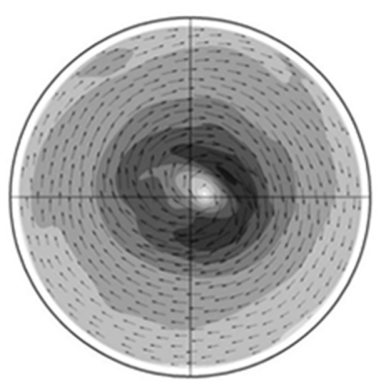

(c)
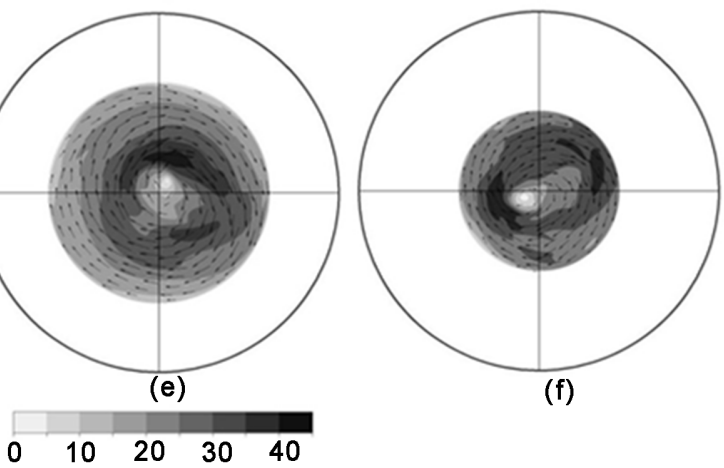

Figure 5. Contour plots of the LES velocity magnitude in six horizontal cross sections: (a) $Z=1.875 D$, (b) $Z=1.5 D$, (c) $Z=1.125 D$, (d) $Z=0.75 D$, (e) $Z=0.375 D$, and (f) $Z=0$.

The simulated results of mean and fluctuating tangential and axial velocities from four turbulence models are compared with experiments. The non-dimensional velocity profiles are scaled with the inlet velocity $\left(U_{i n}=12.68\right.$ $\left.\left(\mathrm{m} \cdot \mathrm{s}^{-1}\right)\right)$. Based on the mesh convergence study, the medium mesh was first used for the SST and SSG-RSM models. However, since the position of the first node for the SST model should be set at a location of $y^{+}<2$, the simulations with the medium grid led to divergence due to the large aspect factor in the mesh. Hence, the fine grid (or mesh (4)) was instead applied for the SST model to obtain a converged solution. Furthermore, as adequately high resolution is a necessity for LES, the finest mesh (mesh (5)) was used in this case to exclude uncertainties connected to the mesh.

The experimental and simulated velocity profiles with different turbulence models are illustrated in Figure 6. The experimental mean tangential velocity profiles are generally captured well by all of the turbulence models. The peak of average tangential velocity and the width of the vortex core region agree properly.

However, as expected, the $\mathrm{k}-\varepsilon$ model predicts a too wide vortex core, and so it has trouble to predict the location of peak tangential velocity (esp. more clear in the section II-II). In addition, this model fails to reproduce the mean axial velocity in all positions. In particular, it predicts mean axial velocities to be uniform in the inner region of the flow where the other models and LDA shows that the mean axial velocity profile has a significant dip creating a central part of 

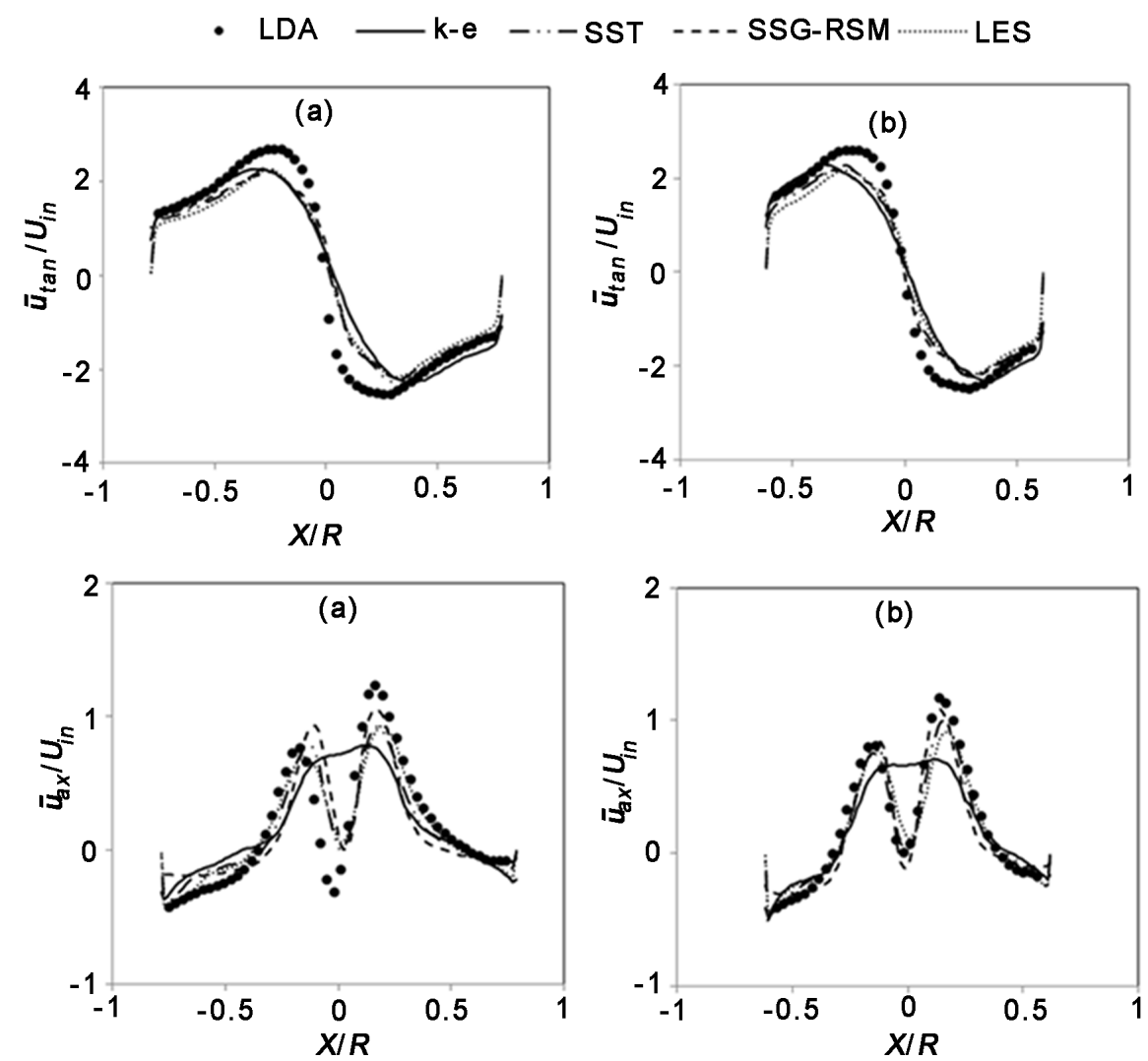

Figure 6. Comparison between velocity profiles for LDA measurement and the simulated time-averaged tangential and axial velocities at two sections on the $X-Z$ plane $(Y=0)$ : (a) section I-I, (b) section II-II.

the profile that resembles a flattened " $M$ ". The asymmetric M-shape of axial velocity profiles is caused by axial pressure gradients that are dictated by axial development of the tangential velocity. Therefore, prediction of axial velocity is more complex and harder than tangential velocity. However, for the two other RANS models and the LES approach, mean axial velocity results agrees well with measurements with a clearly visible M-profile.

The SST model also predicts the asymmetry of the profiles well. This implies that the boundary layer flow close to the wall has a significant effect on the core region of the flow, since the main difference between the two eddy viscosity models lies in the treatment of the near wall region. The SSG-RSM simulation with the medium mesh also yields an impressive agreement of mean axial velocity profiles with experimental data. To start the LES simulation, the solution from the SST simulation was interpolated on the grid of the LES simulation and used as the initial condition to save computational time. However, the LES predicts mean tangential and axial velocities not much better than the RANS models in the two considered positions.

The experimental data from the other important locations of the cyclone, e.g. the cylindrical portion or vortex finder, was not available. Hence, the simulated results cannot be assessed at those locations to give additional information about 
the performance of the models.

On the other hand, as already mentioned, velocity fluctuation levels and the related turbulence dispersion are as significant as mean velocities for the separation process. Turbulent fluctuations enhance particle dispersion, while mean velocities (especially tangential velocity) determine the centrifugal force and the average residence time of the particle in the cyclone. Root-mean square (RMS) values of the tangential and axial velocities from all simulations except the results of $k-\varepsilon$ model are shown and compared to LDA results in Figure 7. The eddy viscosity models do not predict the fluctuations directly; therefore, the fluctuation levels are evaluated based on the diagonal components (normal stresses). The measurements show a peak in the fluctuation levels in the core region due to the PVC and relatively low RMS values in the outer region.

Very large gradients of tangential velocity near the center lead to the high fluctuation levels. They typically are twice or thrice as high as the fluctuations in the outer region. In this respect, the LES distinguishes itself from the RANS models and gives a much better prediction of the velocity fluctuations than these models. The SST model totally fails to predict the peak in the center region, whereas the SSG-RSM agrees qualitatively well with the measurements except in the narrow peak region. Similar comments can be applied to the axial RMS velocities. Overall, the LES performs well while the RANS models are incapable to predict the fluctuations correctly.
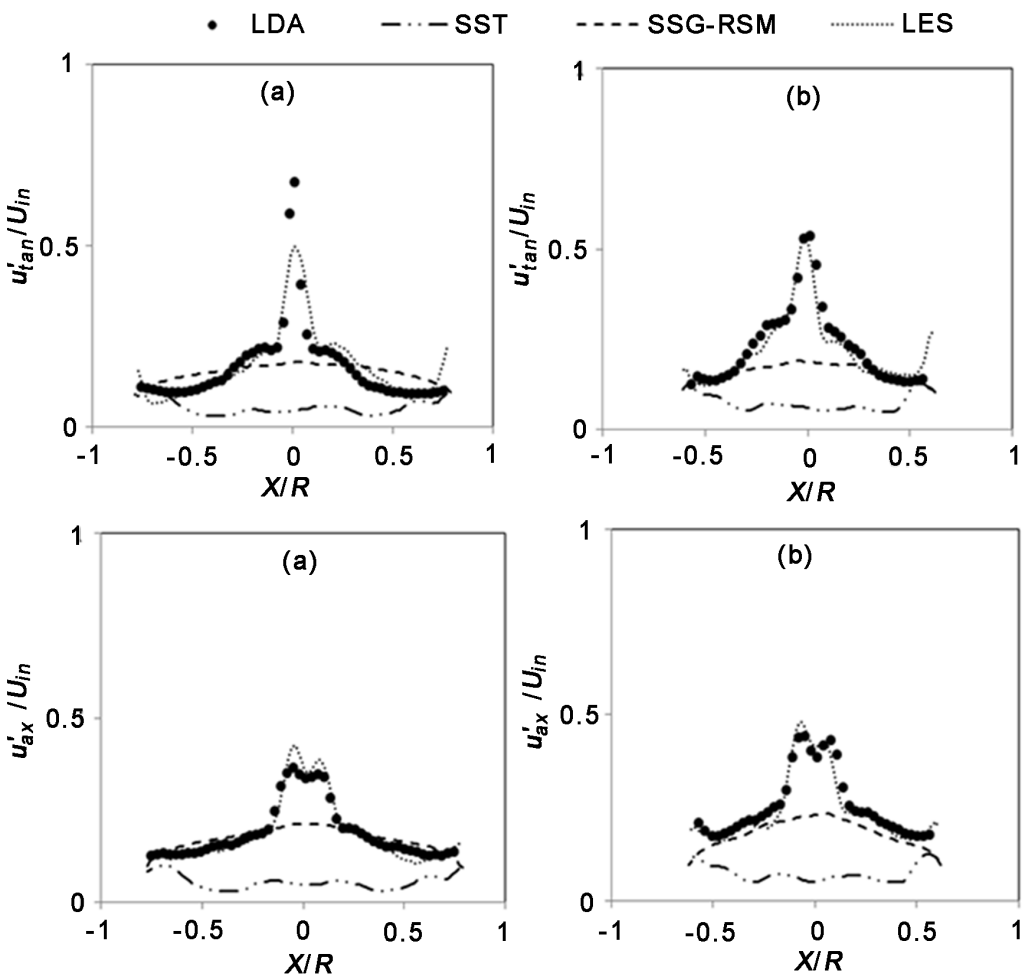

Figure 7. Comparison between velocity profiles for LDA measurement and the simulated RMS values of tangential and axial velocities at two sections on the $X$ - $Z$ plane ( $Y=0)$ : (a) section I-I, (b) section II-II. 
In conclusion, as expected, the LES approach is the best to represent the effects of turbulent dispersion from the velocity fluctuations. The motions of large eddies in the vicinity of the centerline, which are mainly responsible for anisotropic behavior of the flow, can be simulated directly in LES, while the effect of the smaller eddies are captured by SGS models. Thus, since the flow is predominantly governed by the large eddies rather than small eddies, especially in the central region, a large eddy approach can reveal more of the flow physics in the cyclone geometry. Nevertheless, the negative feature of the LES simulations is the requirement of a much finer computational grid, leading to a higher computational effort. Therefore, based on the results in the conical section, the time-averaged results of SST and RSM-SSG models agree well with the experimental results and are very similar to those in the LES, while they are unable to predict the fluctuations due to the PVC. However, the SSG-RSM model showed that it could work even with coarse grids, so it took less time to accomplish a simulation relative to the SST model. A larger number of mesh elements had to be used for the SST model, so it was more time consuming than the SSG-RSM model. Hence, the SSG-RSM model seems to be the model with the best cost-performance ratio.

For modeling a cyclone gasifier including reactant particles, although the SST and the SSG-RSM models are incapable of capturing the PVC phenomenon in the core region, they both can be used for an engineering modeling. In a cyclone gasifier, there is a hypothesis that the gasification process occurs at the wall and smaller unconverted solid particles in addition to soot and tar moves toward the outlet at the top [8]. Thus, it is likely that the PVC phenomenon doesn't influence the gasification process substantially. The SSG-RSM can be used as a first priority due to its ability to work with the coarser grids which results in less computational time. The SST model due to its sensitivity to the near wall grid point needs a finer grid compared to the SSG-RSM leading to a higher computational time. However, adding heat and mass transfer will definitely change the situation, and probably creates new problems. However, based on the recent studies, the SST model is more popular for combustive and thermal flows than the SSG-RSM [46] [47]. On the other hand, the SSG-RSM is the most used industrial model for simulating a cyclone separator [19] [48]. Accordingly, determination of the suitable turbulence model for the final design depends on the adaptability of the reactant and heat transfer equations to the turbulence models.

\section{Conclusions}

The single phase flow of a gas cyclone separator with tangential inlet was simulated with four different turbulence models and all but the k- $\varepsilon$ model could be validated against LDA measurement from literature [29]. The four turbulence models are 1) standard $\mathrm{k}-\varepsilon$ model with curvature correction; 2) SST model with curvature correction; 3) SSG Reynolds stress model and 4) LES with Smagorinsky sub-grid model. 
For the mean tangential velocities, all RANS models and the LES captured the shape of the tangential velocity profiles with good accuracy; nevertheless, the $k-\mathcal{E}$ model predicted a much wider central region than the experiments and the other simulated results. For the mean axial velocity the situation is more complicated. The SST model with its integration through the boundary layer all the way to $y^{+}<2$ agrees much better with the results than the $k$ - $\mathcal{E}$ model, which was unable to predict the $\mathrm{M}$-shaped axial velocity profile. The mean axial velocity results with the SSG-RSM model were also capable of capturing the quasi-periodic fluctuating asymmetric results from the LDA experiments. However, for the results from the LES simulations, no significant difference in comparison to the RANS-based simulations was observed.

Due to the precessing vortex core, velocity fluctuation levels are quite high near the central region. The RMS values of fluctuations from the experiments were compared to the velocity fluctuations of the simulations. While the RANS models are incapable to predict this phenomenon accurately, the LES simulation gives an excellent agreement with the experiments.

Moreover, the simulations demonstrated a peak in radial velocity just beneath the vortex finder entrance, caused by the lip leakage flow. There are no LDA measurements from this region but lip leakage is a well-known phenomenon in industrial cyclones and it is important that the models can predict it.

Overall, the LES approach was the most accurate one, but also the most time-consuming. However, the SST model required a grid with a larger number of elements to resolve the boundary layers close to the walls while the SSG-RSM model uses a wall function that requires less grid points close to the wall. For this reason, the SST model became more time-consuming and the SSG-RSM model seems to be the best alternative for engineering design where computational cost/performance is of primary importance. However, making a decision for choosing the most appropriate turbulence model for the final design of a gasification process in a cyclone depends on the behavior of the reactant and heat transfer equations against the turbulence models.

\section{Acknowledgements}

The research was performed under financial support provided by the Bio4 Gasification and Bio4 Energy collaborations. The authors would also like to express their gratitude to Prof. Gernot Staudinger and Dr. Stefan Obermair for providing LDA measurement data.

\section{References}

[1] Risberg, M., Öhrman, O.G.W., Gebart, B.R., Nilsson, P.T., Gudmundsson, A. and Sanati, M. (2014) Influence from Fuel Type on the Performance of an Air-Blown Cyclone Gasifier. Fuel, 116, 751-759. https://doi.org/10.1016/j.fuel.2013.08.008

[2] Gabra, M., Pettersson, E., Backman, R. and Kjellström, B. (2001) Evaluation of Cyclone Gasifier Performance for Gasification of Sugar Cane Residue-Part 1: Gasification of Bagasse. Biomass and Bioenergy, 21, 351-369. 
https://doi.org/10.1016/S0961-9534(01)00043-5

[3] Gabra, M., Pettersson, E., Backman, R. and Kjellströ6m, B. (2001) Evaluation of Cyclone Gasifier Performance for Gasification of Sugar Cane Residue-Part 2: Gasification of Cane Trash. Biomass and Bioenergy, 21, 371-380. https://doi.org/10.1016/S0961-9534(01)00044-7

[4] Zhao, Y., Sun, S., Che, H., Guo, Y. and Gao, C. (2012) Characteristics of Cyclone Gasification of Rice Husk. International Journal of Hydrogen Energy, 37, 16962-16966. https://doi.org/10.1016/j.ijhydene.2012.08.093

[5] Guo, X.J., Xiao, B., Zhang, X.L., Luo, S.Y. and He, M.Y. (2009) Experimental Study on Air-Stream Gasification of Biomass Micron Fuel (BMF) in a Cyclone Gasifier. Bioresource Technology, 100, 1003-1006. https://doi.org/10.1016/j.biortech.2008.07.007

[6] Zhao, Y., Sun, S., Zhang, T. and Zhou, H. (2013) Experimental Research on Fuel Staging Cyclone Gasification of Wood Powder. Fuel, 103, 53-57. https://doi.org/10.1016/j.fuel.2011.08.020

[7] Risberg, M., Carlsson, P. and Gebart, R. (2015) Numerical Modeling of a $500 \mathrm{~kW}$ Air-Blown Cyclone Gasifier. Applied Thermal Engineering, 90, 694-702. https://doi.org/10.1016/j.applthermaleng.2015.06.056

[8] Risberg, M. (2013) Entrained Flow Gasification of Biomass: On Atomisation, Transport Processes and Gasification Reactions. Ph.D. Thesis, Luleå University of Technology, Luleå.

[9] Barth, W. (1955) Der Einfluß Der Vorgänge in Der Grenzschicht Auf Die Abscheideleistung von Mechanischen Staubabscheidern, Staubbewegungen in Der Grenzschicht. VDIBer, 6, 20-32.

[10] Muschelknautz, E. and Brunner, K. (1967) Untersuchungen an Zyklonen. Chemie Ingenieur Technik-CIT, 39, 531-538. https://doi.org/10.1002/cite.330390908

[11] Hoffmann, A. and Stein, L. (2008) Gas Cyclones and Swirl Tubes: Principles, Design and Operation. Springer-Verlag, Berlin, Heidelberg.

[12] Boysan, F., Ayers, W.H. and Swithenbank, J. (1982) A Fundamental Mathematical Modelling Approach to Cyclone Design. Chemical Engineering Research and Design, 60a, 222-230.

[13] Meier, H.F. and Mori, M. (1999) Anisotropic Behavior of the Reynolds Stress in Gas and Gas-Solid Flows in Cyclones. Powder Technology, 101, 108-119. https://doi.org/10.1016/S0032-5910(98)00162-4

[14] Minier, J., Simonin, O. and Gabillard, M. (1991) Numerical Modelling of Cyclone Separators. Proceedings of the International Conference on Fluidized Bed Combustion, Quebec.

[15] Hoekstra, A.J., Derksen, J.J. and Van Den Akker, H.E.A. (1999) An Experimental and Numerical Study of Turbulent Swirling Flow in Gas Cyclones. Chemical Engineering Science, 54, 2055-2065. https://doi.org/10.1016/S0009-2509(98)00373-X

[16] Shukla, S.K., Shukla, P. and Ghosh, P. (2011) Evaluation of Numerical Schemes for Dispersed Phase Modeling of Cyclone Separators. Engineering Applications of Computational Fluid Mechanics, 5, 235-246. https://doi.org/10.1080/19942060.2011.11015367

[17] Derksen, J.J., Van den Akker, H.E.A. and Sundaresan, S. (2008) Two-Way Coupled Large-Eddy Simulations of the Gas-Solid Flow in Cyclone Separators. AIChE Journal, 54, 872-885. https://doi.org/10.1002/aic.11418

[18] Dhakal, T.P., Walters, D.K. and Strasser, W. (2014) Numerical Study of Gas-Cyclone 
Airflow: An Investigation of Turbulence Modelling Approaches. International Journal of Computational Fluid Dynamics, 28, 1-15. https://doi.org/10.1080/10618562.2013.878800

[19] Gronald, G. and Derksen, J.J. (2011) Simulating Turbulent Swirling Flow in a Gas Cyclone: A Comparison of Various Modeling Approaches. Powder Technology, 205, 160-171. https://doi.org/10.1016/j.powtec.2010.09.007

[20] Misiulia, D., Andersson, A.G. and Lundström, T.S. (2015) Computational Investigation of an Industrial Cyclone Separator with Helical-Roof Inlet. Chemical Engineering \& Technology, 38, 1425-1434. https://doi.org/10.1002/ceat.201500181

[21] Shalaby, H., Wozniak, K. and Wozniak, G. (2008) Numerical Calculation of Particle-Laden Cyclone Separator Flow Using Les. Engineering Applications of Computational Fluid Mechanics, 2, 382-392. https://doi.org/10.1080/19942060.2008.11015238

[22] Schlichting, H. and Gersten, K. (2000) Boundary-Layer Theory. Springer, Berlin. https://doi.org/10.1007/978-3-642-85829-1

[23] Shepherd, C.B. and Lapple, C.E. (1939) Flow Pattern and Pressure Drop in Cyclone Dust Collectors. Industrial \& Engineering Chemistry, 31, 972-984.

https://doi.org/10.1021/ie50356a012

[24] Peng, W., Hoffmann, A., Boot, P.J.A., Udding, A., Dries, H.W., Ekker, A. and Kater, J. (2002) Flow Pattern in Reverse-Flow Centrifugal Separators. Powder Technology, 127, 212-222. https://doi.org/10.1016/S0032-5910(02)00148-1

[25] Yazdabadi, P.A., Griffiths, A.J. and Syred, N. (1994) Characterization of the PVC Phenomena in the Exhaust of a Cyclone Dust Separator. Experiments in Fluids, 17, 84-95. https://doi.org/10.1007/BF02412807

[26] Hoekstra, A.J., Vliet, E., Derksen, J.J. and Akker, H.E.A. (1998) Vortex Core Precession in a Gas Cyclone. Advances in Turbulence VII, 289-292.

https://doi.org/10.1007/978-94-011-5118-4_71

[27] Smith, J.L. (1962) An Experimental Study of the Vortex in the Cyclone Separator. Journal of Basic Engineering, 84, 602. https://doi.org/10.1115/1.3658721

[28] Derksen, J.J. and Van den Akker, H.E.A. (2000) Simulation of Vortex Core Precession in a Reverse-Flow Cyclone. AIChE Journal, 46, 1317-1331.

https://doi.org/10.1002/aic.690460706

[29] Obermair, S., Woisetschläger, J. and Staudinger, G. (2003) Investigation of the Flow Pattern in Different Dust Outlet Geometries of a Gas Cyclone by Laser Doppler Anemometry. Powder Technology, 138, 239-251. https://doi.org/10.1016/j.powtec.2003.09.009

[30] Ferziger, J. H. and Perić, M. (2002) Computational Methods for Fluid Dynamics. Springer Berlin Heidelberg, Berlin, Heidelberg. https://doi.org/10.1007/978-3-642-56026-2

[31] Launder, B.E. and Spalding, D.B. (1974) The Numerical Computation of Turbulent Flows. Computer Methods in Applied Mechanics and Engineering, 3, 269-289. https://doi.org/10.1016/0045-7825(74)90029-2

[32] Menter, F.R. (1994) Two-Equation Eddy-Viscosity Turbulence Models for Engineering Applications. AIAA Journal, 32, 1598-1605. https://doi.org/10.2514/3.12149

[33] Spalart, P.R. and Shur, M. (1997) On the Sensitization of Turbulence Models to Rotation and Curvature. Aerospace Science and Technology, 1, 297-302. https://doi.org/10.1016/S1270-9638(97)90051-1

[34] ANSYS CFX-Solver Theory Guide (2013) Release 14.5. ANSYS Inc., Canonsburg. 
[35] Launder, B.E., Reece, G.J. and Rodi, W. (1975) Progress in the Development of a Reynolds-Stress Turbulence Closure. Journal of Fluid Mechanics, 68, 537-566. https://doi.org/10.1017/S0022112075001814

[36] Speziale, C.G., Sarkar, S. and Gatski, T.B. (2006) Modelling the Pressure-strain Correlation of Turbulence: An Invariant Dynamical Systems Approach. Journal of Fluid Mechanics, 227, 245. https://doi.org/10.1017/S0022112091000101

[37] Wilcox, D.C. (1998) Turbulence Modeling for CFD. 2nd Edition, DCW Industries, La Canada, California.

[38] Shalaby, H.H. (2006) On the Potential of Large Eddy Simulation to Simulate Cyclone Separators. Ph.D., Thesis, Chemnitz University of Technology, Chemnitz.

[39] Schmitt, F.G. (2007) About Boussinesq's Turbulent Viscosity Hypothesis: Historical Remarks and a Direct Evaluation of Its Validity. Comptes Rendus Mécanique, 335, 617-627. https://doi.org/10.1016/j.crme.2007.08.004

[40] Smagorinsky, J. (1963) General Circulation Experiments with the Primitive Equations. Monthly Weather Review, 91, 99-164. https://doi.org/10.1175/1520-0493(1963)091<0099:GCEWTP>2.3.CO;2

[41] Lilly, D.K. (1992) A Proposed Modification of the Germano Subgrid-Scale Closure Method. Physics of Fluids A: Fluid Dynamics, 4, 633.

https://doi.org/10.1063/1.858280

[42] Germano, M., Piomelli, U., Moin, P. and Cabot, W.H. (1991) A Dynamic Subgrid-Scale Eddy Viscosity Model. Physics of Fluids A: Fluid Dynamics, 3, 1760. https://doi.org/10.1063/1.857955

[43] Sagaut, P. (2006) Large Eddy Simulation for Incompressible Flows. Springer-Verlag, Berlin, Heidelberg.

[44] Ferziger, J. and Peric, M. (2013) Computational Methods for Fluid Dynamics. Springer-Verlag, Berlin, Heidelberg.

[45] ANSYS CFX-Solver Modeling Guide (2013) Release 14.5. ANSYS Inc., Canonsburg.

[46] Musa, O., Xiong, C., Changsheng, Z. and Lunkun, G. (2016) Assessment of the Modified Rotation/curvature Correction SST Turbulence Model for Simulating Swirling Reacting Unsteady Flows in a Solid-Fuel Ramjet Engine. Acta Astronautica, 129, 241-252. https://doi.org/10.1016/j.actaastro.2016.09.016

[47] Musa, O., Changsheng, Z., Xiong, C. and Lunkun, G. (2016) Prediction of Swirling Cold Flow in a Solid-Fuel Ramjet Engine with a Modified Rotation/Curvature Correction SST Turbulence Model. Applied Thermal Engineering, 105, 737-754. https://doi.org/10.1016/j.applthermaleng.2016.03.091

[48] Cortés, C. and Gil, A. (2007) Modeling the Gas and Particle Flow inside Cyclone Separators. Progress in Energy and Combustion Science, 33, 409-452. https://doi.org/10.1016/j.pecs.2007.02.001 
Symbols Used

\begin{tabular}{|c|c|c|}
\hline$a$ & {$[\mathrm{~m}]$} & cyclone inlet height \\
\hline$A_{\text {in }}$ & {$\left[\mathrm{m}^{2}\right]$} & area of the cyclone inlet surface \\
\hline$A_{\text {out }}$ & {$\left[\mathrm{m}^{2}\right]$} & area of the cyclone outlet surface \\
\hline$b$ & {$[\mathrm{~m}]$} & cyclone inlet width \\
\hline$C_{P}$ & {$[-]$} & pressure loss coefficient \\
\hline$C_{\mu}$ & {$[-]$} & $k$ - $\varepsilon$ based model constant \\
\hline$D$ & {$[\mathrm{~m}]$} & cyclone body diameter \\
\hline$D_{b}$ & {$[\mathrm{~m}]$} & cyclone dust bin diameter \\
\hline$D_{d}$ & {$[\mathrm{~m}]$} & cyclone drop tube diameter \\
\hline$D_{x}$ & {$[\mathrm{~m}]$} & cyclone vortex finder diameter \\
\hline$f_{r}$ & {$[-]$} & $\begin{array}{l}\text { rotation function as a multiplier of eddy viscosity turbulent } \\
\text { production term }\end{array}$ \\
\hline$H$ & {$[\mathrm{~m}]$} & total cyclone height \\
\hline$H_{b}$ & {$[\mathrm{~m}]$} & cyclone dust bin height \\
\hline$H_{c}$ & {$[\mathrm{~m}]$} & cyclone cone height \\
\hline$H_{d}$ & {$[\mathrm{~m}]$} & cyclone drop tube height \\
\hline$k$ & {$\left[\mathrm{~m}^{2} \cdot \mathrm{s}^{-2}\right]$} & turbulent kinetic energy \\
\hline$L_{V}$ & {$[\mathrm{~m}]$} & cyclone vortex finder length \\
\hline $\bar{p}$ & {$[\mathrm{~Pa}]$} & time-averaged static pressure \\
\hline$\overline{\bar{p}}$ & {$[\mathrm{~Pa}]$} & filtered static pressure \\
\hline$\Delta p$ & {$[\mathrm{~Pa}]$} & pressure drop \\
\hline$P_{i n}$ & {$[\mathrm{~Pa}]$} & cyclone inlet pressure \\
\hline$p_{k}$ & {$\left[\mathrm{~Pa} \cdot \mathrm{s}^{-1}\right]$} & turbulent production term in eddy viscosity models \\
\hline$P_{\text {out }}$ & {$[\mathrm{Pa}]$} & cyclone outlet pressure \\
\hline $\begin{array}{l}=s g s \\
S_{i j}\end{array}$ & {$\left[\mathrm{~s}^{-1}\right]$} & large-scale strain rate tensor \\
\hline$t$ & {$[\mathrm{~s}]$} & time \\
\hline$u$ & {$\left[\mathrm{~m} \cdot \mathrm{s}^{-1}\right]$} & fluid velocity \\
\hline$u^{\prime}$ & {$\left[\mathrm{m} \cdot \mathrm{s}^{-1}\right]$} & fluctuating fluid velocity \\
\hline $\bar{u}$ & {$\left[\mathrm{~m} \cdot \mathrm{s}^{-1}\right]$} & mean (time-averaged) fluid velocity \\
\hline$\overline{\bar{u}}$ & {$\left[\mathrm{~m} \cdot \mathrm{s}^{-1}\right]$} & filtered fluid velocity \\
\hline$U_{\text {in }}$ & {$\left[\mathrm{m} \cdot \mathrm{s}^{-1}\right]$} & cyclone inlet normal speed \\
\hline
\end{tabular}

\section{Greek Letters}

\begin{tabular}{ccc}
$\delta$ & {$[-]$} & Kronecker delta \\
$\varepsilon$ & {$\left[\mathrm{m}^{2} \cdot \mathrm{s}^{-3}\right]$} & dissipation rate \\
$\mu_{t}$ & {$[\mathrm{~Pa} \cdot \mathrm{s}]$} & eddy viscosity \\
$v$ & {$\left[\mathrm{~m}^{2} \cdot \mathrm{s}^{-1}\right]$} & kinematic viscosity \\
$v^{\text {sgs }}$ & {$\left[\mathrm{m}^{2} \cdot \mathrm{s}^{-1}\right]$} & SGS viscosity \\
$\rho$ & {$\left[\mathrm{kg} \cdot \mathrm{m}^{-3}\right]$} & fluid density \\
$\rho_{g}$ & {$\left[\mathrm{~kg} \cdot \mathrm{m}^{-3}\right]$} & gas density \\
$\tau_{i j}^{s g s}$ & {$\left[\mathrm{~m}^{2} \cdot \mathrm{s}^{-2}\right]$} & SGS stress \\
$\omega$ & {$\left[\mathrm{s}^{-1}\right]$} & specific dissipation rate \\
\hline
\end{tabular}


Abbreviations

\begin{tabular}{cc}
\hline LES & large eddy simulation \\
PVC & precessing vortex core \\
RANS & Reynolds-averaged Navier-Stokes \\
RSM & Reynolds stress model \\
SGS & subgrid-scale \\
SST & shear stress transport \\
WALE & wall-adapted local eddy viscosity \\
\hline
\end{tabular}

\section{Scientific Research Publishing}

Submit or recommend next manuscript to SCIRP and we will provide best service for you:

Accepting pre-submission inquiries through Email, Facebook, LinkedIn, Twitter, etc. A wide selection of journals (inclusive of 9 subjects, more than 200 journals)

Providing 24-hour high-quality service

User-friendly online submission system

Fair and swift peer-review system

Efficient typesetting and proofreading procedure

Display of the result of downloads and visits, as well as the number of cited articles Maximum dissemination of your research work

Submit your manuscript at: http://papersubmission.scirp.org/

Or contact eng@scirp.org 